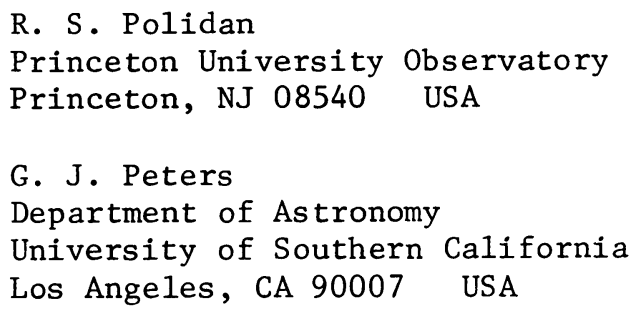

Abstract. In this paper, we present far ultraviolet observations of three interacting binary stars (HR'2142, HR 7084, and $\lambda$ Tauri). All stars exhibit evidence of a gas stream and extensive mass outflow from the system. Physical parameters are derived for the gas stream in HR 2142 .

The physical conditions in the gas streams in close binary systems produce the strongest spectral features in the far ultraviolet. This has made direct study of the mass flow difficult until the advent of satellite astronomy. Observations of three active systems (Table 1 ) were obtained with the Copernicus satellite during the past two years.

Table 1

Observations

\begin{tabular}{l|c|c|c|c}
\hline Star & Components & P(Days) & $\mathrm{M}_{2} / \mathrm{M}_{1}$ & Dates of Observation \\
HR 2142 & BlVe+gK: & 80.86 & $\sim 0.1$ & Dec. 76-Feb. 79 \\
HR 7084 & B3Ve+F-K & 6.70 & $\sim 0.2$ & Aug. 78- May 79 \\
$\lambda$ Tau & B3IV+gA & 3.95 & 0.26 & Feb. 78 \\
\hline
\end{tabular}

Selected spectral features between $1000 \mathrm{~A}$ and $1400 \mathrm{~A}$ were investigated at high resolution $(0.05 \mathrm{~A})$ in HR 2142 and $\lambda$ Tauri and at low $(0.20 \mathrm{~A})$ resolution in HR 7084. Details of the equipment used and technique of observation can be found in Rogerson et al. (1973).

293

M. J. Plavec, D. M. Popper and R. K. Ulrich (eds.), Close Binary Stars: Observations and Interpretation, $293-297$. Copyright $\odot 1980$ by the IAU. 
HR 2142

$\mathrm{HR} 2142$ was suggested to be a mass transfer binary by Peters and Polidan (1973) on the basis of ground based observations. The binary model was refined (Peters 1976) with more observations suggesting a binary with a permanent accretion disk, a thick gas stream, and a counterstream. The ultraviolet observations were obtained at twelve phase points over seven orbital cycles.

Strong, variable absorption lines, consistent with the existence of a gas stream, were seen in C II, N II, Si II, Si III, S II, S III and Fe III. The gas stream was not detected in lines of $\mathrm{C} \mathrm{I}, \mathrm{C}$ III, $\mathrm{N}$, $\mathrm{N}$ III, O I, and Fe II. Figure 1 shows the phase dependence of the gas stream line strengths normalized to the strength at phase 0.95 . The points connected by the solid line was obtained from ground based observations of the hydrogen Balmer lines (Peters 1976). This curve was found to fit the strength variation of the lower ionization (Si II and S II) UV lines. The points with error bars represent the strength variation of the higher ionization species (C II, N II, Si III, S III, and Fe III). Figure 2 shows one of these lines, Fe III 1130.404, at three phases: 0.7 (no stream), 0.95 (peak stream), and 0.02 (counter-stream).

The radial velocity variation of the gas stream lines is shown in Figure 3. Again, the points connected by the solid line were obtained from analysis of ground based data and the low ionization UV lines. The points with error bars represent the velocities of the higher ionization species in the gas stream.

Analysis of the ultraviolet line strengths, velocities, and shapes has yielded three principal results. The gas stream is quite complex. At least two major, repeatable from cycle to cycle, components exist: one producing the higher ionization species and one responsible for the lower ionization species. The slower variation of the higher ionization lines suggests that they are formed in a more extended region than the low ionization lines. Both components appear to be composed of many (>3)

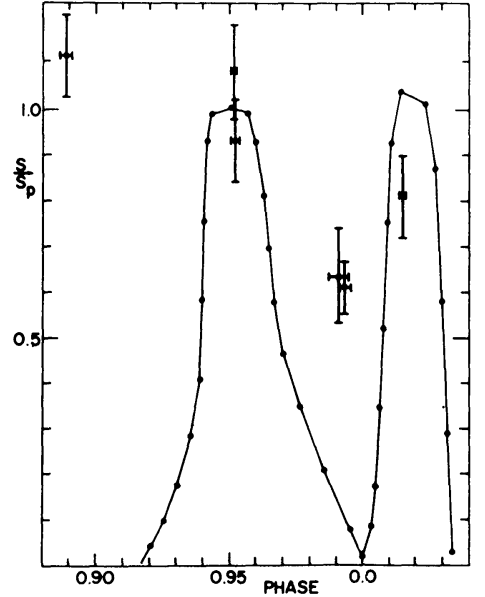

Figure 1. Phase dependence of the gas stream lines in HR 2142 .

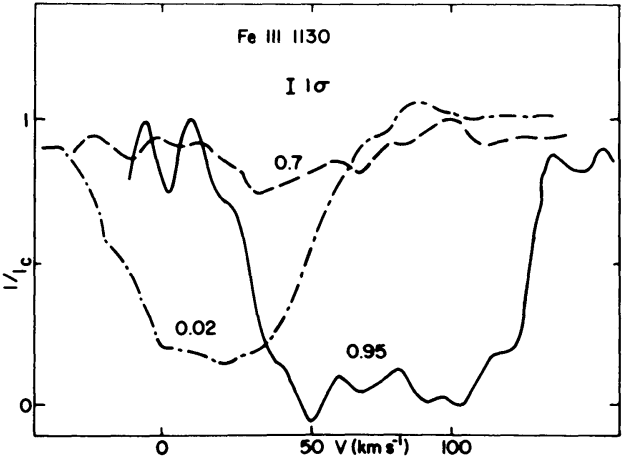

Figure 2. Fe III 1130.404 at three phases in HR 2142. 

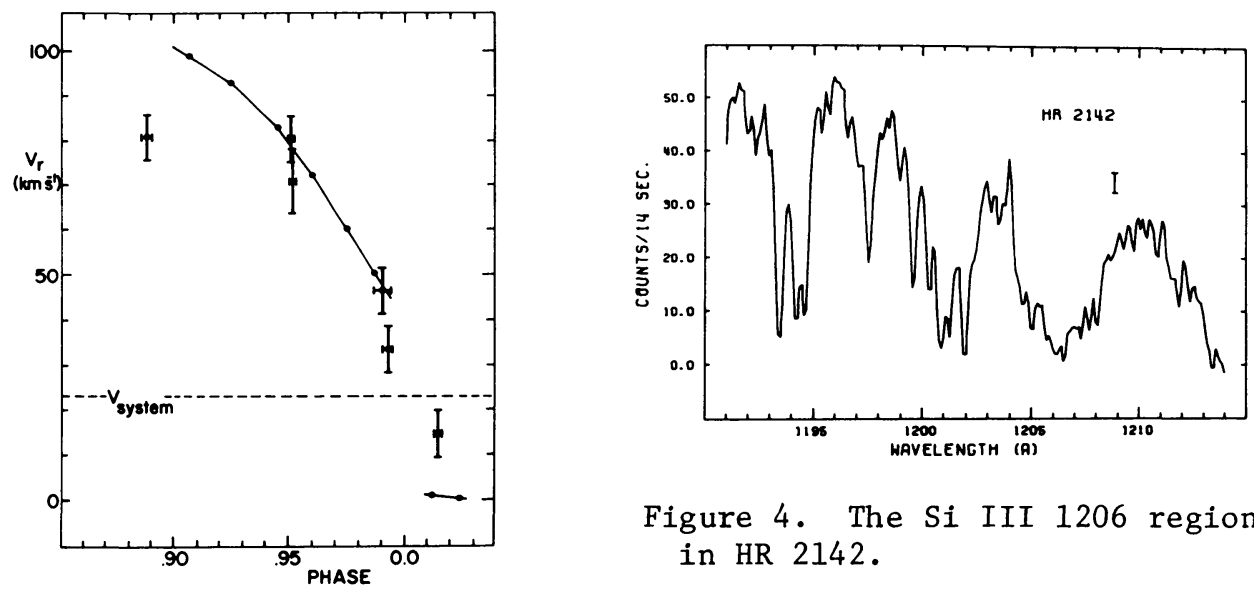

Figure 4. The Si III 1206 region in HR 2142.

Figure 3. Radial velocity versus phase for the gas stream lines in HR 2142.

discrete "clouds" that do not repeat from cycle to cycle. Analysis of the principal "cloud" in each component suggests that in the higher ionization zone $\mathrm{Te} \sim 14000 \mathrm{~K}$ and $\mathrm{N}_{\mathrm{e}} \sim 1012 \mathrm{~cm}-3$ and in the lower ionization zone $\mathrm{T}_{\mathrm{e}} \sim 11000 \mathrm{~K}$ and $\mathrm{N}_{\mathrm{e}} \sim 1013 \mathrm{~cm}-3$. These conditions suggest that the gas stream has a high density "core" and a hot, lower density "halo". The physical conditions discussed above and the model presented earlier (Peters 1976) imply a minimum mass transfer rate of $10^{-7} \mathrm{M}_{\odot} \mathrm{yr}^{-1}$ in this system. The final piece of information obtained from the analysis regards the angle at which the gas stream crosses the system. The observed velocity variation (Figure 3) argues in favor of a much greater angle with respect to the line of centers than is predicted by theory (Lubow and Shu 1975). The velocity field is, however, complex. Further analysis and more observation will be required before this deviation can be confirmed.

Observations of HR 2142 at phases when the gas stream is not visible suggest that most of the material that leaves the secondary is ejected from the system and not accreted. In particular, near phase 0.5 negatively displaced $\left(\mathrm{V} \sim 100 \mathrm{~km} \mathrm{~s}^{-1}\right)$ absorption components, comparable in strength to the strongest gas stream lines are observed. Also, despite the impinging gas stream the rapidly rotating primary star displays a reasonably strong stellar wind at Si III 1206 (Figure 4). Evidence of mass ejection from the system can also be seen in C II 1335 where moderately strong, sharp absorption components not associated with the interstellar medium are seen. The most probable origin of these components is an extensive circumsystem disk or cloud.

Summarizing the results for HR 2142, the physical parameters derived for the gas stream are within the range predicted from theoretical calculations (cf. Lubow and Shu 1975) with the possible exception of the angle the stream makes with the line of centers. Extensive mass outflow from the binary is observed, suggesting that at this stage of evolution little material is being accreted by the primary star in HR 2142 . 
HR 7084

This Be star has been shown to be an interacting binary star by Koubsky (1978). The ultraviolet observations of this system are less extensive and of lower resolution than those of HR 2142. The general behavior of HR 7084 in the ultraviolet is quite similar to that of HR 2142. A prominent gas stream is seen between phases 0.8 and conjunction and an extensive outflow of material is seen near phase 0.5 . The physical conditions in the stream are similar to those found for HR 2142 . A strong stellar wind is also seen in the Si III 1206 line of the primary star.

\section{$\lambda$ Tauri}

The eclipsing binary star $\lambda$ Tauri has been briefly surveyed at high resolution. A gas stream was dectected but at a substantially reduced strength compared to HR 2142 and HR 7084. Similarly, only weak evidence was found for the existence of mass outflow from the system. However, numerous ( 3 to 6 ) weak, non-interstellar absorption components were found in C II, N I, and N II, suggesting the presence of multiple circumsystem disks or clouds.

This work was supported by NASA grants NAS 5-23576 (RSP) and NSG 5356 (GJP).

\section{REFERENCES}

Koubsky, P.: 1978, Bul1. Astron. Inst. Czechos1., 29, pp. 288.

Lubow, S. and Shu, F.: 1975, Astroph. J., 198, pp. 383.

Peters, G. J.: 1976, in Be and Shell Stars (Ed. A. Slettebak), IAU Symp. No. 70 , pp. 417.

Peters, G. J. and Polidan, R. S.: 1973, in Extended Atmospheres and Circumstellar Matter in Spectroscopic Binary Systems, IAU Symp. No. 51, pp. 174.

Rogerson, J. B., Spitzer, L., Drake, J. F., Dressler, K., Jenkins, E., Morton, D. C., and York, D. G.: 1973, Astroph. J., 181, pp. L97.

\section{DISCUSSION FOLLOWING POLIDAN AND PETERS}

Shore: In light of the wind blowing from the B star, have you looked for $\mathrm{X}$-ray emission from the inflowing matter slamming into the stellar wind? It could be a soft source.

Polidan: On the Copernicus satellite there is a co-aligned X-ray proportional counter operated by the University College, London. Throughout the ultraviolet observations of these systems X-ray data were accumulated. In no case was a significant X-ray flux detected. 
The $\mathrm{X}$-ray detector is sensitive only in the 3-8 keV range, however, so the presence of soft $\mathrm{X}$-rays cannot be ruled out.

Shore: What is the velocity of the wind?

Polidan: The wind velocity is typical of that found in single rapidly rotating stars--a few hundred $\mathrm{kms}^{-1}$.

Ha11: Two questions about HR 2142: First, you said that no mass is assimilated, that all left the system. One hundred per cent loss would have far-reaching implications for the theory of mass loss but $\sim 50 \%$ loss, which would be easier to explain would involve only a factor 2 in your derivation of $\dot{M}$ from the observations. Can the observational determination of really be so precise? Second, would it be worthwhile to monitor it photometrically, say, in UBV for several years.

Polidan: At this time we can say with a fair degree of confidence that more than $50 \%$, possibly as much as $90 \%$, of the transferred material is lost from the environment of the components of the binary. With the completion of our detailed analysis the fraction of the transferred material that is lost will be better established. The weaker unsaturated lines in the UV allow a very accurate determination of the relative column densities of the stream and outflow. We do believe that the observations are of sufficient quality that as little as $10 \%$ accretion could be detected.

It would be very worthwhile to photometrically monitor HR 2142 . The star is almost certainly variable in light. The existence of 1 ight variations, periodic or non-periodic, would help in understanding the mass transfer in this system. Similarly, I would like to encourage polarimetric observations of this system over its 81 day period. Also, the other system that I mentioned, HR 7084, would benefit greatly from photometric and polarimetric observations. 